Bangladesh J. Bot. 42(2): 273-278, 2013 (December)

\title{
AEROMYCOFLORA OF THE DHAKA UNIVERSITY CAMPUS
}

\author{
Juglul Ahmed, KS Hossain ${ }^{1}$ and MA Bashar \\ Department of Botany, University of Dhaka, Dhaka-1000, Bangladesh
}

Key words: Aeromycoflora, Dhaka University campus

\begin{abstract}
In an average, 2106 fungal colony forming units were settled within ten minutes on one square meter area at noon from the air of Dhaka University campus during February 2011 to January 2012. Among the identified fungi, Aspergillus was one of the most dominating genus in all the stations over the study period. The second was Penicillium followed by Cladopsorium, Curvularia, Alternaria, Fusarium, Trichoderma, Pestalotia, Rhizopus and Colletotrichum. In the dry winter (December - February), Alternaria, Cladosporium, Curvularia and Rhizopus showed its peak. Hot humid summer (April) is the most favourable season for the occurrence of Colletotrichum. Similarity has been found in fungal biodiversity in the indoor and outdoor air. However, higher number of colony was recorded from indoor $(57.23 \%)$ than that of outdoor air.
\end{abstract}

\section{Introduction}

The atmosphere of earth contains air borne viruses, bacteria, protozoa, pollen grains, different propagules and vegetative cells of algae, fungi, lichens, bryophytes and pteridophytes. Among these, fungal spores play a significant role in childhood asthma, allergies, mycotoxicity, biodeterioration and infections of man and animals (Burge 1985, Aimanianda et al. 2010). Airborne fungi are considered to act as indicator of the level of atmospheric bio-pollution (Kakde et al. 2001). About $20 \%$ of the human population is easily sensitized by normal fungal spore concentrations (up to $10^{6}$ spores $/ \mathrm{m}^{3}$ ) and all fungal spores should be regarded as potentially allergenic. Numerous plant diseases such as rusts, smuts, mildews, leaf spots, etc. are caused by air borne fungi (Kendrick 2000).

Occurrence of fungal spores in the air varied season to season remarkably because of variation of weather conditions. Warm and dry weather favours the development, sporulation and dispersal of conidia of Cladosporium, Epicoccum and Alternaria and the greatest daily concentration of conidia of these genera usually occurs at noon and after noon. It is also varied with vegetation types under and around the study area. The greatest concentrations of Alternaria spores were noted at the harvesting time (Chakraborty et al. 2003, Stepalska and Jerzy 2005, Kasprzyk 2008).

Surveys on these aspects have been made in different countries of the world following impaction or sedimentation method ( $\mathrm{Li}$ and Kendrick 1995, Khan et. al. 1999, Ianovici and Tudorica 2009, Sharma 2011). The sedimentation method is still quite popular in India and some other countries. The method is cheap and simple and is also recommended by Polish Standards (Fleischer et al. 2006, Sekulska et al. 2007, Sharma 2011).

In Bangladesh, study on aerobiology and air borne bio-particle has been done (Khan and Alio 1984 and Pasha and Hossain 2011). But no investigation on air borne fungi has been carried out particularly in the Dhaka metropolitan where 14.5 million people breathe. The present investigation has therefore, been undertaken to study the monthly distribution pattern of mycoflora in the air of the Dhaka University campus.

${ }^{1}$ Author for correspondence: Department of Botany, Jagannath University, Dhaka, Bangladesh $<$ ksh1968@gmail.com> 


\section{Materials and Methods}

Ten different locations of the Dhaka University Campus $\left(23^{\circ} 42^{\prime} 0^{\prime \prime} \mathrm{N}\right.$ and $\left.90^{\circ} 22^{\prime} 30^{\prime \prime} \mathrm{E}\right)$ were selected for the sampling of air borne fungi. Among these locations five were inside the buildings of Arts Faculty, University Medical Centre, Science Library, Shahidullah Hall, Mycology and Plant Pathology Laboratory of the Botany Department. Remaining five locations were at the open areas of Mol Chottor, Hakim Chottor, Teachers Students Centre, Mukarram Bhaban and Botanical Garden. In this investigation, gravity plate sampling method (Sharma 2011) was followed for isolation of air borne fungi at the selected locations.

Sampling was done monthly at noon from February 2011 to January 2012 excluding the months of October and December. Three culture media viz. Czapek's Dox Agar (CDA), Potato Dextrose Agar (PDA) and Emmons version of Sabouraud Agar (SA) media were used (Emmons et al. 1977). During the selected day of each month, about $20 \mathrm{ml}$ sterile culture media were poured onto each pair of Petri plates ( $9 \mathrm{~cm}$ in dia.). To cheek bacterial contamination, a drop of $50 \%$ lactic acid was added with each plate under laminar air flow. Each pair of Petri plates were sealed with paraffin strip. At each selected location, nine Petri plates containing sterile culture media ( 3 plates for each medium) were exposed horizontally for ten minutes on a $1.5 \mathrm{~m}$ high tripod stand. After that, the exposed Petri plates were sealed with paraffin strip and taken into the laboratory and incubated at $25 \pm 2^{\circ} \mathrm{C}$ for five days. The fungal colonies developed on the culture media were examined and identified with the help of standard mycological books and manuals.

Per cent abundance and frequency of the fungal colonies was calculated by adopting the formula of Pathak 2012. Temperature and relative humidity of the selected months at the sampling sites were determined by a digital Hygro-thermometer machine (Mextech-1). Monthly precipitation of the Dhaka city was collected from the official website of Bangladesh Meteorological Department.

\section{Results and Discussion}

Only viable fungal spores or mycelial fragments of saprophytes and facultative parasites those settled on culture media were formed colonies. A total of 12068 colonies were recorded from the 900 culture plates exposed for ten minutes from the ten sampling points. Nine hundred culture plates make $5.73 \mathrm{~m}^{2}$ sampling area, therefore, on an average, ca. 2106 colony forming units (CFU) per square meter were settled during ten minutes exposure. Out of this, $219(1.82 \%)$ colonies were sterile mycelia (Table 1). Sharma (2011) reported that Mycelia sterilia were $9.19 \%$ of the total fungi settled on PDA media at the selected tea garden during the summer. Some fungi need specific culture medium and/or physical stimulation for spore formation and some others are rigorously non-spore forming. Moreover, fungal sporulation highly depends on weather condition. Consequently, fewer non-spore forming fungi were also found in the present study.

Table 1 shows that the spore forming colonies were distributed into ten genera viz. Alternaria, Aspergillus, Cladosporium, Colletotrichum, Curvularia, Fusarium, Penicillium, Pestalotia, Rhizopus and Trichoderma under the class Zygomycetes and Deuteromycetes. In accordance with Sharma (2011), the member of Ascomycetes and Basidiomycetes were totally absent and anamorphic fungal genera were recorded as a dominant fungal group.

All the fungi recorded in Table 1 were grown on the three culture media used in the present investigation, none was absent on any culture media. Their abundance on the different culture media, however, varied. The highest number of colony was found on CDA (4253) followed by PDA (4172) and SA (3642). Per cent abundance of the fungi on these three media reveals that Aspergillus, Cladosporium, Alternaria, Curvularia and Pestalotia were preferred to grow on 
CDA, whereas, Penicillium, Trichoderma and Rhizopus were on PDA and Fusarium and Colletotrichum were on SA.

Among the identified fungi Aspergillus was one of the most dominating genera at all the locations and months in the air of the campus (Table 1). Its collective per cent abundance was 42.60 on the three nutrient media followed by Penicillium (27.61), Cladopsorium (8.49), Curvularia (4.76), Alternaria (4.65), Fusarium (3.25), Trichoderma (2.56), Pestalotia (2.04) and Rhizopus (1.36). The abundance of Colletotrichum was the lowest (0.87). This result is in agreement with similar variations that have been reported from India (Sharma 2011). Following gravity plate method, highest per cent abundance of Aspergillus (41.35) in the air of Darjeeling tea garden followed by Mucor (10.34), Penicillium (9.19), Rhizopus (8.04), Trichoderma (5.74), Curvularia (3.44), Nigrospora (2.29), and Cladopsorium (1.14) was observed. The variation in aeromycoflora and its abundance with the present study might be due to the difference of weather condition and vegetation between the study areas.

Table 1. Air borne fungi on three culture media during February, 2011 to January, 2012 at the Dhaka University campus.

\begin{tabular}{|c|c|c|c|c|c|}
\hline \multirow[t]{2}{*}{ Fungal genera } & \multicolumn{3}{|c|}{$\begin{array}{l}\text { Collective number of fungal colonies on three } \\
\text { culture media }(*)\end{array}$} & \multirow{2}{*}{$\begin{array}{l}\text { Total } \\
\text { colony }\end{array}$} & \multirow{2}{*}{$\begin{array}{c}\text { Frequency } \\
(\%)\end{array}$} \\
\hline & $\mathrm{CDA}^{*}$ & PDA & SA & & \\
\hline Alternaria & $247(2.05) * *$ & $186(1.54)$ & $128(1.06)$ & $561(4.65)$ & 28.89 \\
\hline Aspergillus & 1853(15.36) & $1693(14.04)$ & $1594(13.21)$ & 5141 (42.60) & 100.00 \\
\hline Cladosporium & 394 (3.27) & $352 \quad(2.92)$ & $277(2.30)$ & $1023(8.49)$ & 46.67 \\
\hline Colletotrichum & $35(0.29)$ & $32 \quad(0.26)$ & $39(0.32)$ & $105(0.87)$ & 7.78 \\
\hline Curvularia & $227(1.88)$ & 185 (1.53) & $164(1.36)$ & $576(4.76)$ & 31.11 \\
\hline Fusarium & $47(0.39)$ & $140 \quad(1.16)$ & 205 (1.70) & $392(3.25)$ & 22.22 \\
\hline Penicillium & $1116(9.25)$ & $1237(10.26)$ & $977(8.10)$ & 3331 (27.61) & 100.00 \\
\hline Pestalotia & $99(0.82)$ & $56(0.46)$ & $92(0.76)$ & $247(2.04)$ & 14.44 \\
\hline Rhizopus & $51(0.42)$ & $60(0.50)$ & $53(0.44)$ & $164(1.36)$ & 12.22 \\
\hline Trichoderma & $106(0.88)$ & $123(1.02)$ & $80(0.66)$ & $309(2.56)$ & 18.89 \\
\hline Sterile mycelia & $78(0.65)$ & $108(0.90)$ & $33(0.27)$ & 219 (1.82) & 14.44 \\
\hline Total colonies & 4253(35.25) & 4172 (34.58) & 3642 (30.17) & $12068(100)^{* * *}$ & \\
\hline
\end{tabular}

*CDA = Czapek's Dox Agar, PDA = Potato Dextrose Agar and SA = Sabouraud Agar. **Per cent abundance of fungal colonies within parenthesis. ${ }^{* *}$ Total culture plates used $=900\left(5.73 \mathrm{~m}^{2}\right)$, number of CFU settled $=$ $2,106 / \mathrm{m}^{2}$.

Pathak (2012) from Madhya Prodesh, India found abundance of Aspergillus and Penicillium which were 32 and $9 \%$, respectively by using particle sampler. The abundances of these two fungi were also quite higher in the present investigation. Sedimentation method does not permit exact quantitative determination. Some earlier observations reported that results of sedimentation method are usually higher than numbers obtained with the use of air samplers (Fleischer et al. 2006). However, data collected by sedimentation method allow the drawing of correct conclusions on types of fungi present in the air and can give a rough approximation of fungal concentration.

Table 1 also showed that Aspergillus and Penicillium were found in the highest per cent frequency (100) followed by Cladosporium (46.67), Curvularia (31.11), Atlernaria (28.89), Fusarium (22.22), Trichoderma (18.89), Pestalotia (14.44), Rhizopus (12.22) and Colletotrichum (7.78). Pathak (2012) recorded Aspergillus in the highest per cent frequency (95.85) followed by Penicillium (84.80), Atlernaria (83.30), Cladosporium (54.15), Curvularia (41.70), Rhizopus 
(41.66), Fusarium (39.15), Mucor (33.35), Epicoccum (33.3), Phoma (29.15), Nigrospora (21.35) and Trichoderma (16.65). The variation in aeromycoflora and its frequencies with the present study might be due to the variation of meteorological parameters of the study areas and methods used in the investigations.

Tables 2 and 3 show variations in sedimentation of fungi on the three culture media among seasons and locations. Number of colony of five fungal genera viz. Aspergillus, Fusarium, Penicillium, Pestalotia and Trichoderma showed its peak and near to peak during warm rainy monsoon (June - September). Among them Aspergillus, Penicillium and Pestalotia found at its peak in indoor location. Whereas, two others found at its peak in outdoor location. Alternaria, Cladosporium, Curvularia and Rhizopus showed its peak and near to peak during dry winter (December - February) and Colletotrichum found its peak in hot humid summer (April).

Table 2. Monthly total fungal colonies of air borne fungi on three culture media and climatic factors during February, 2011 to January, 2012 at 10 different sampling sites of the Dhaka University campus.

\begin{tabular}{lrrrrrrrrrr}
\hline \multirow{2}{*}{ Fungal genera } & \multicolumn{10}{c}{ Collective number of air borne fungi in ten different months } \\
\cline { 2 - 11 } & Feb & Mar & April & May & June & July & Aug & Sep & Dec & Jan \\
\hline Alternaria & $\mathbf{1 0 4}$ & 62 & 58 & 42 & 18 & 33 & 25 & 40 & 95 & 84 \\
Aspergillus & 123 & 193 & 335 & 509 & 624 & 714 & 843 & $\mathbf{1 0 7 2}$ & 379 & 349 \\
Cladosporium & $\mathbf{2 0 4}$ & 80 & 73 & 61 & 61 & 67 & 53 & 50 & 198 & 162 \\
Colletotrichum & 7 & 18 & $\mathbf{3 3}$ & 13 & 10 & 7 & 6 & 0 & 5 & 6 \\
Curvularia & 89 & 65 & 33 & 47 & 27 & 19 & 37 & 24 & $\mathbf{1 3 6}$ & 99 \\
Fusarium & 25 & 28 & 30 & 35 & 46 & 57 & $\mathbf{7 3}$ & 62 & 23 & 13 \\
Penicillium & 313 & 254 & 219 & 209 & 250 & 339 & 411 & $\mathbf{5 4 6}$ & 347 & 443 \\
Pestalotia & 33 & 25 & 10 & 0 & 0 & 0 & $\mathbf{1 0 9}$ & 12 & 11 & 47 \\
Rhizopus & 25 & 11 & 0 & 11 & 7 & 5 & 7 & 15 & $\mathbf{4 7}$ & 36 \\
Trichoderma & 19 & 21 & 0 & 15 & 22 & 57 & $\mathbf{8 9}$ & 63 & 11 & 12 \\
Sterile mycelia & 33 & 22 & 18 & 12 & 0 & 0 & $\mathbf{7 9}$ & 11 & 14 & 30 \\
\hline Total colonies & 975 & 786 & 809 & 954 & 1065 & 1298 & 1732 & 1895 & 1266 & 1288 \\
\hline Relative humidity (\%) & 54.0 & 47.8 & 72.8 & 72.0 & $\mathbf{8 1 . 1}$ & 61.6 & 63.3 & 72.2 & 52.4 & 57.1 \\
Temp. $\left({ }^{\circ} \mathrm{C}\right)$ & 24.3 & 27.5 & 29.2 & 31.1 & $\mathbf{3 1 . 7}$ & 27.1 & 26.9 & 31.5 & 21.3 & 20.2 \\
Precipitation (mm) & 0.0 & 6.8 & 8.6 & 6.3 & 15.5 & 12.7 & 15.1 & $\mathbf{1 8 . 4}$ & 0.0 & 7.0 \\
\hline
\end{tabular}

Cladosporium is the fungal genera most correlated with meteorological parameters. This may be attributed to the size and nature of conidia. Cladosporium produces dry conidia in chains easily carried through air. Therefore, dispersion of Cladosporium spores is more influenced by meteorological parameters than Alternaria spores (Awad 2005). In accordance with the present study, Levetin (1995) reported that members of dry-air spores (Cladosporium, Alternaria and Curvularia) were found in greatest abundance in the atmosphere characterised by low humidity, generally during warmer afternoon hours.

Table 3 shows that fungi which were dominant in the indoor air were also recorded in significant concentration in the outdoor air and vice versa. The table also shows that similarity has been existed in fungal biodiversity in the indoor and outdoor air. This is in agreement with the report from India (Kotwal et al. 2010). In comparison to 5 outdoor sampling sites, higher contribution $(57.23 \%)$ were found in 5 indoor sampling sites. In accordance with the present investigation Sekulska et al. (2007) also reported that amount of fungi was higher in the indoor than that of outdoor air. 


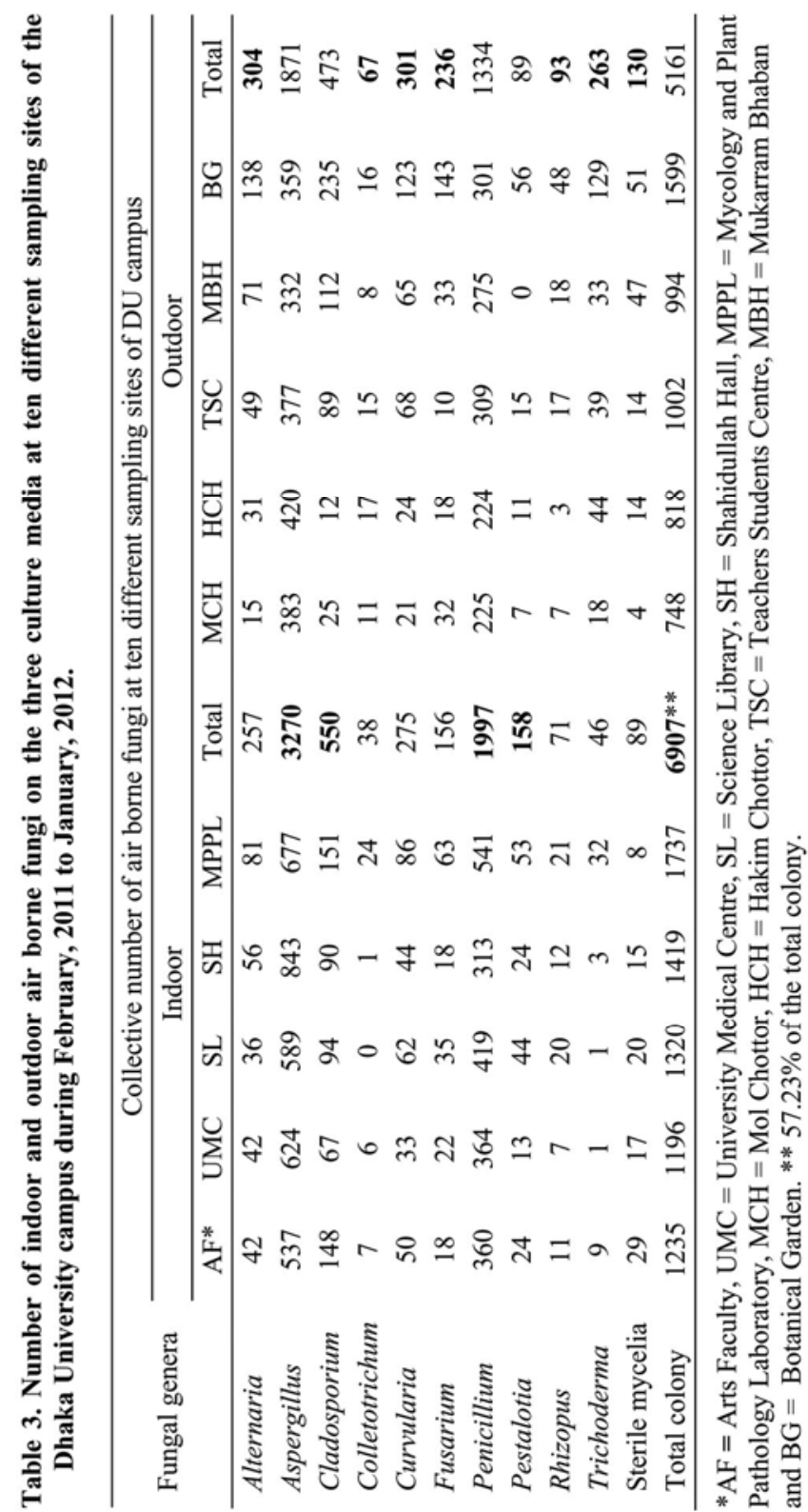


Among the fungi, found in the present investigation, Atlernaria, Aspergillus, Cladosporium, Curvularia, Fusarium, Penicillium and Rhizopus were reported as pathogenic to plants and/or human and strongly allergenic to human. Colletotrichum and Pestalotia were reported as only plant pathogenic (Burge 1985, Kendrick 2000, Kotwal et al. 2010). The present study contributes to our knowledge of airborne spores in the Dhaka city. Regular monitoring of airborne fungi can be helpful in the prevention of fungal allergic diseases in the city.

\section{References}

Aimanianda V, Bayrz J, Bozza S, Kniemeyer O and Perruccio K 2010. Clever cloak prevents immune recognition of air borne fungal spores. $4^{\text {th }}$ advances against Aspergillosis, Asp. Newsl. 460: 1117-1123.

Awad A 2005. Vegetation: A source of air fungal bio-contaminant. Aerobiol. 21: 53-61.

Burge HA 1985. Fungus allergens. Clin. Rev. Allergy 3: 319-329.

Chakraborty PS, Gupta-Bhattacharya and Chanda S 2003. Aeromycoflora of an agricultural farm in West Bengal, India. Grana. 42: 248-254.

Emmons CW, Binford CH, Utz JP and Kwon-Chung KJ 1977. Medical Mycology, 3rd ed., Lea \& Febiger, Philadelphia, PA. pp. 535.

Fleischer RM, Bober-Gheek B, Bortkiewcz O and Rusiecka-Ziolkowska J 2006. Microbiological control of airborne contamination in hospitals. Indoor and Built Environ. 15(1): 53-56.

Ianovici N and Tudorica D 2009. Aeromycoflora in outdoor environment of Timisoara City (Romania). Not. Sci. Biol. 1(1): 21-28.

Kakde UB, Kakde HU and Saoji AA 2001. Seasonal variation of fungal propagules in a fruit market environment, Nagpur (India). Aerobiologia 17: 177-182.

Kasprzyk I 2008. Aeromycology- main research fields of interest during the last 25 years. Ann. Agric. Environ. Med. 15: 1-7.

Kendrick B 2000. The fifth kingdom. $3^{\text {rd }}$ ed. Focus Publishing, R. Pullins Co. Newburyport MA 01950, USA. pp. XI+373.

Khan MR and Alio S 1984. An aerobiological study of the Dhaka City and its sub-urban areas. Bangladesh. J. Bot. 13(2): 214-219.

Khan ZU, Khan MAY, Chady R and Sharma PN 1999. Aspergillus and other moulds in the air of Kuwait. Mycopathol. 146: 25-32.

Kotwal SG, Gosavi SV and Deore KD 2010. Aeromycoflora of outdoor and indoor air of residential area in Nashik. Asian J. Exp. Biol. Sci. SPL.: 24-30.

Levetin E 1995. Fungi. p. 87-120, In: Ianovici N and D Tudorica 2009. Aeromycoflora in outdoor environment of Timisoara City (Romania). Not. Sci. Biol. 1(1): 21-28.

Li De-Wei and Kendrick B 1995. A year-round comparison of fungal spores in indoor and outdoor air. Mycologia. 87(2): 190-195.

Pasha MK and Hossain MS 2011. Airborne bio-particulate objects at Chittagong University campus. Bang. J. Bot. 40(2): 189-191.

Pathak K 2012. An extramural aeromycological investigation of dental college hospital associated environment. Int. J. Env. Sci. 2(4): 1952-1961

Sekulska M, Stryjakowska, Piotraszewska-Pajak A, Szyszka A, Nowicki M and Filipiak M 2007. Microbiological quality of indoor air in university room. Polosh J. Env. Stud. 16(4): 623-632.

Sharma K 2011. Comparative study of aeromycoflora in relation to soil mycoflora of Darjeeling tea garden, India. Recent Research in Science and Technology 3(5): 84-86.

Stepalska D and Jerzy W 2005. Variation in fungal spore concentrations of selected taxa associated to weather conditions in Cracow, Poland in 1997. Aerobiol. 21: 43-52. 